\title{
Study of visual outcome in patients undergoing penetrating keratoplasty
}

\author{
Vaishali $^{{ }^{*} \text {, Kavita Kumar }}{ }^{2}$, Bhavana Sharma ${ }^{3}$ \\ Dept. of Ophthalmology, Gandhi Medical College, Bhopal, Madhya Pradesh, India
}

\section{Article Info}

Received: 30 th June, 2019

Accepted: $6^{\text {th }}$ July, 2019

Published Online: $9^{\text {th }}$ September, 2019

Keywords: Corneal vascularisation, Graft failure, Penetrating keratoplasty.

\begin{abstract}
Introduction: To study the visual outcome in patients undergoing penetrating keratoplasty (PK).

Materials and Methods: This was a prospective interventional study concerning patients who underwent optical and therapeutic PK in Hamidia hospital Bhopal during a period of Dec 2016 to May 2108. Age, gender, eye, indication of PKP, and bestcorrected visual acuity (BCVA) constituting the preoperative data were recorded in a predesigned proforma. Penetrating keratoplasty was performed using similar technique and patients were followed up till a period of 1 year and post operative complications were identified and managed accordingly. The outcome was assessed in terms of percentage improvement in logMAR visual acuity and the variables affecting the final visual outcome were studied.

Results: Of the total 50 cases of penetrating keratoplasty that were analyzed 33 were done for therapeutic purposes and 17 were done for optical reasons. Mean recipient age was 39.88 years \pm 2.9 years, range $3-68$. Follow up period was 1 year. The indications were therapeutic (34\%), post traumatic or post infective corneal scarring $(50 \%)$, previous graft failure $(6 \%)$ and pseudophakic bullous keratopathy $(4 \%)$. In optical PK, $62.5 \%$ recipient eyes showed good visual improvement (improvement of more than -0.8 $\log$ MAR). $21.8 \%$ eyes showed moderate visual improvement, and $15.6 \%$ eyes showed no visual improvement. In eyes with therapeutic PK, $46.7 \%$ showed good visual improvement, $13.3 \%$ moderate and $40 \%$ showed no visual improvement.

Conclusion: Penetrating keratoplasty done for both optical and therapeutic indications resulted in fairly good visual outcome. The pre-operative host risk factors were associated with an increased probability of graft rejection. Careful workup of recipients for corneal transplantation with reference to local and systemic risk factors is of utmost importance in the outcome of corneal transplantation with regards to graft survival.
\end{abstract}

\section{Introduction}

Corneal blindness is one of the most challenging public health problems all over the world, especially in developing countries like India. According to WHO, corneal blindness is the $4^{\text {th }}$ leading cause of blindness globally $(5.1 \%) .{ }^{1}$ It is expected that the number of individuals with unilateral corneal blindness in India will inpcrease to 10.6 million by $2020 .^{2}$ The aetiology encompasses ocular trauma, infectious keratitis, bullous keratopathy, corneal degenerations, dystrophies, xerophthalmia, and trachoma. Penetrating keratoplasty $(\mathrm{PK})$ is increasingly providing promising results in visual improvement and preserving the structural integrity of eye in cases of corneal diseases. PK is a fullthickness corneal transplant procedure. In India, the major indications for $\mathrm{PK}$ remain corneal scarring caused by infection, trauma and malnutrition. A successful outcome of penetrating keratoplasty depends on various donor and host factors, surgical technique and post operative care. A major challenge pertaining to success of PK in India lie in the fact that majority of patients undergoing PK come from low socioeconomic status and are illiterate, which renders it difficult to maintain post operative follow up and care. It is necessary to indentify the complications early and to manage accordingly, as these may cause a poorer final visual outcome and also threaten the graft survival. In the present study, cases undergoing PK are studied with special emphasis on the final visual outcome and quality of life.

\section{Objectives}

1. To study the visual outcome in patients undergoing penetrating keratoplasty in a tertiary care centre in Bhopal.

2. To study the effect of host factors on the final visual outcome of penetrating keratoplasty.

\section{Materials and Methods}

The present study "Study of outcome in patients undergoing Penetrating Keratoplasty." is a hospital based prospective interventional study conducted in department of Ophthalmology, Gandhi Medical College and Associated

\footnotetext{
*Corresponding Author: Vaishali, Dept. of Ophthalmology, Gandhi Medical College Bhopal, Madhya Pradesh, India

Email: drvaishaliophthal@gmail.com

http://doi.org/10.18231/j.ijceo.2019.084
} 
Hamidia Hospital, Bhopal during the years August 2016 August 2018.

\section{Inclusion Criteria}

All the patients registered in cornea clinic and in waiting list for penetrating keratoplasty in the department of Ophthalmology, GMC, Bhopal, with the following corneal pathologies - leucomatous corneal opacity, anterior staphyloma, bullous keratopathy, non healing and Sloughing corneal ulcer, corneal perforation, keratoconus and graft failure.

\section{Exclusion Criteria}

Patients with posterior segment abnormality and those undergoing cosmetic PK were excluded.

Data were collected after written informed consent from patients. Demographic details including name, age, sex, address and occupation were noted. A detailed history of illness was elicited and documented, including diminution of vision, opacity, ocular pain, redness, photophobia, or discharge. Past history included ocular trauma, treatment history and previous ocular surgery. Visual acuity in all cases was taken in Snellen's chart and converted to $\log$ MAR units to provide a common scale for analysis. A detailed ocular examination was carried out in torch light and slit lamp and findings were noted in the preset proforma. Donor cornea was evaluated under diffuse illumination aided with slit lamp, and graded as per the standards of EBAI (Eye Bank Association of India). ${ }^{3}$ Full thickness PK was done by corneal surgeons in eye OT of Hamidia hospital, Bhopal. The technique of trephination, procedure, and suturing was noted. All patients were examined and treated postoperatively and complications were managed accordingly. Visual acuity assessment and Slit lamp examination was done routinely. Graft clarity was graded as: ${ }^{4}$

4+: graft clear, but all details of anterior chamber and iris visible.

3+: graft clear, but some details of anterior chamber and iris obscured.

2+: graft hazy, iris and anterior chamber visible but no details discernible

1+: graft very hazy, iris and anterior chamber just visible.

0 : graft opaque.

All the findings were recorded in the prescribed proforma during the indoor period and thereafter followed up after 1 week. 1 month, 3 months, 6 months and 1 year interval. Patients were advised regarding care and to report earlier to us in case of diminution of vision, pain, and watering and excessive foreign body sensation. In every follow up, examination in detail aided with slit lamp was done and findings were recorded. In each follow up visit, a detailed slit lamp examination was performed and findings were noted under a preset proforma.

Good visual outcome was defined as postoperative vision improvement $\geq-0.8 \log$ MAR compared with preoperative vision, moderate outcome as $0-0.7 \log$ MAR improvement, and no improvement if vision remained same or worsened. ${ }^{5}$

Successful graft in terms of graft clarity was defined as graft clarity $2-4$, and failed graft was defined as graft clarity $0-1 .^{5}$ Graft rejection was defined as presence of keratic precipitates, subepithelial and stromal infiltrates, endothelial rejection line and graft vascularisation. ${ }^{5}$

After completion of the study, data was evaluated with appropriate statistical indices and tests of significance. The statistical tests used were Chi square, unpaired T test, Odd's ratio, Anova test, linear regression analysis and spearman correlation coefficient. Results were considered as significant with a two-sided $P$ value of $<0.05$. Data analysis was performed using SPSS (version 15.0, SPSS, USA).

\section{Results}

\section{Pre Study Variables}

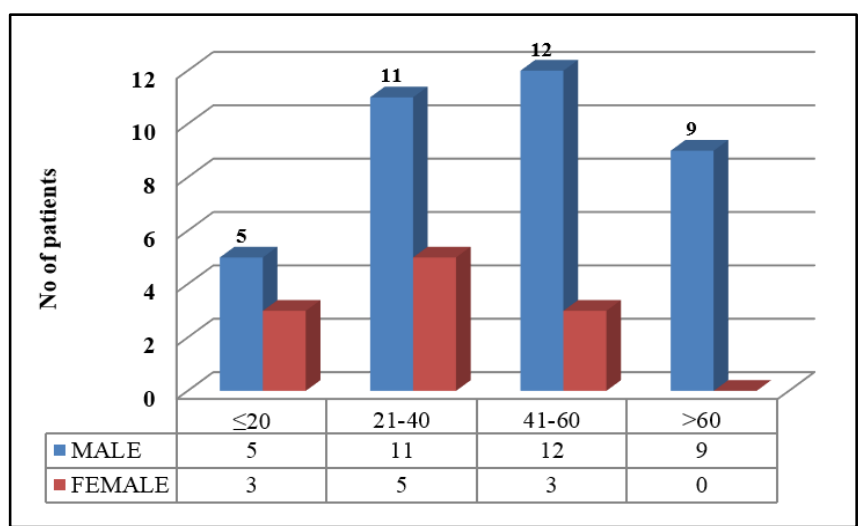

Fig. 1: Age and gender distribution $(n=48)$

The mean age of recipients was 39.88 years \pm 2.9 years SD with a confidence interval of 34.0-45.7 at 95\% CI. Maximum number of recipients $(45.8 \%)$ were in the age group 31-60 years, and minimum, $9(18.8 \%)$ in the age group $>60$ years. $37(77 \%)$ recipients were male while 11 (23\%) were female.

The most common corneal pathology requiring optical PK was leucomatous opacity (26\%), whereas the 2nd most common was adherent Leucoma (20\%). There were 6 eyes (12\%) with graft failure. Among the 16 eyes that underwent therapeutic PK, 14 (28\%) recipients had corneal ulcer and 2 (4\%) had Keratomalacia. 

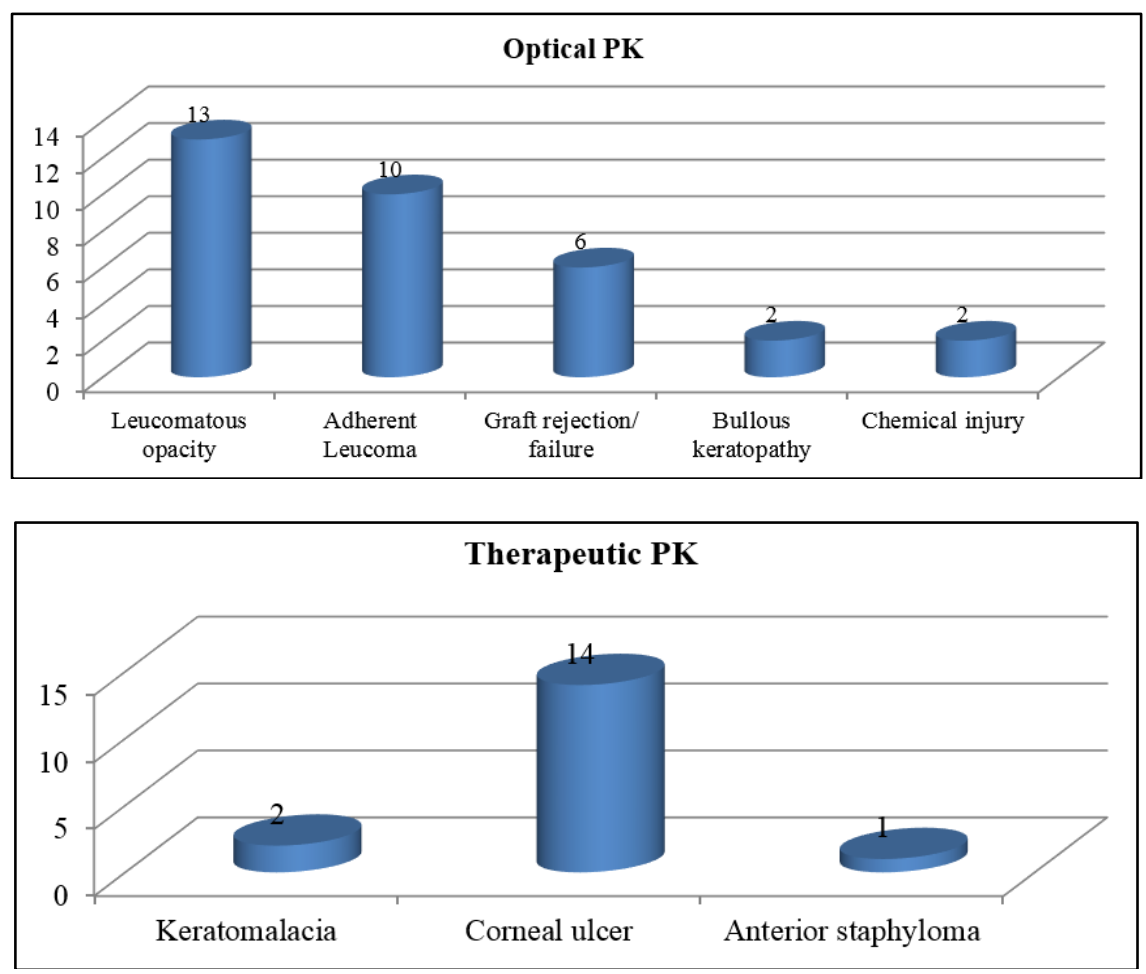

Fig. 2: Distribution of corneal pathologies requiring penetrating keratoplasty $(\mathrm{n}=50)$

\section{Visual Outcome}

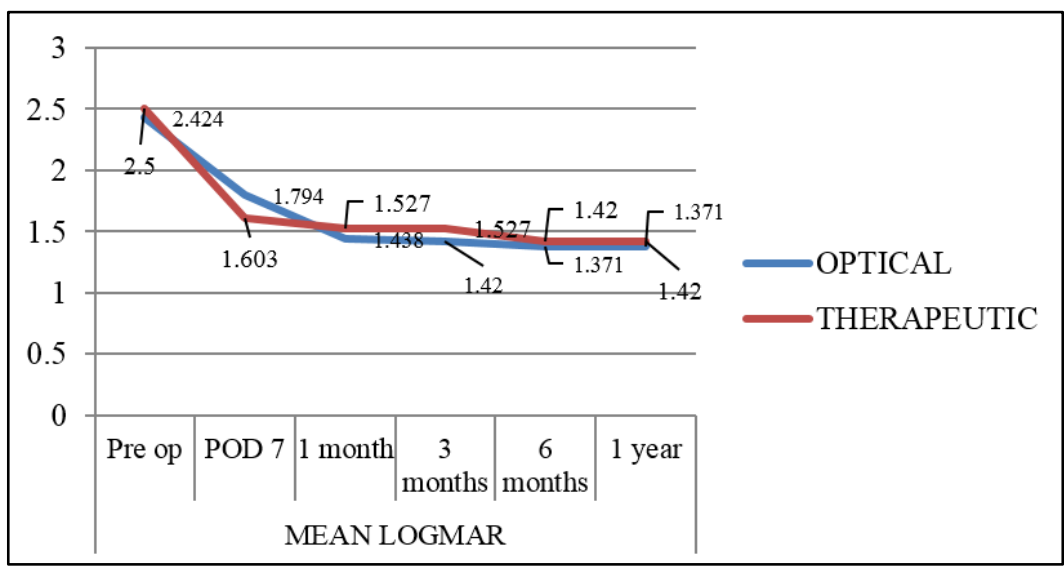

Fig. 3: Comparision between mean logMAR BCVA in optical and therapeutic PK at pre-op and all follow ups

Fig. 3 shows the comparison between the mean visual acuity in $\log$ MAR of optical and therapeutic cases of PK pre-operatively and at all follow ups. Test of significance (unpaired t test) was executed to estimate difference in the final visual outcome between the two groups. The result was insignificant $(>0.5)$, proving that there is no significant statistical difference in the final visual outcome of optical and therapeutic PK.
Table 1: Linear regression analysis with final BCVA as a dependent variable of pre-op BCVA

\begin{tabular}{|c|c|c|c|c|}
\hline Model & $\mathbf{R}$ & $\mathbf{R}^{\mathbf{2}}$ & Anova F & Significance 'P' \\
\hline 1 & 0.249 & 0.062 & 18.902 & 0.046 \\
\hline
\end{tabular}

Table 1 demonstrates the BCVA 1 year post-operative in direct relationship with the pre-operative BCVA. Linear regression analysis was executed to estimate the linear relationship between final visual outcome as a dependant variable of pre-operative BCVA. It shows that pre-operative $\mathrm{BCVA}$ is a predictor of final visual outcome with a variance of $45 \%$. 


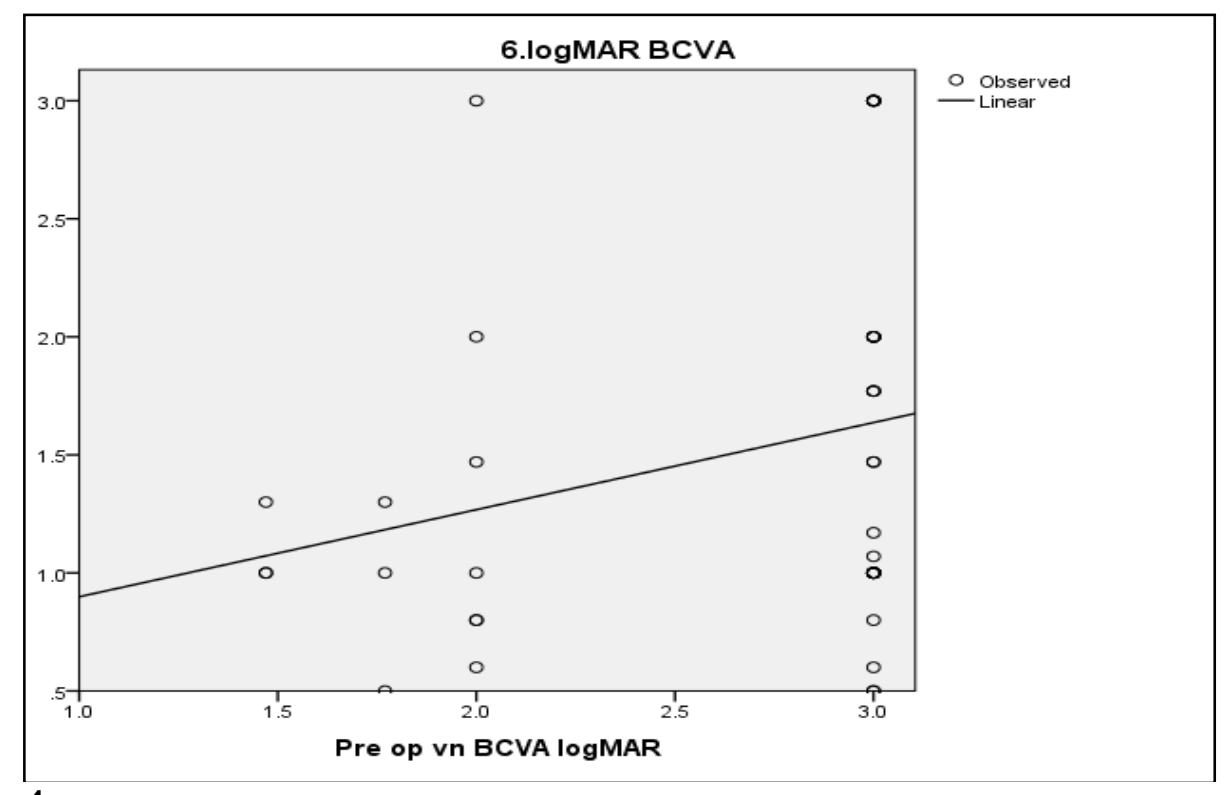

Fig. 4

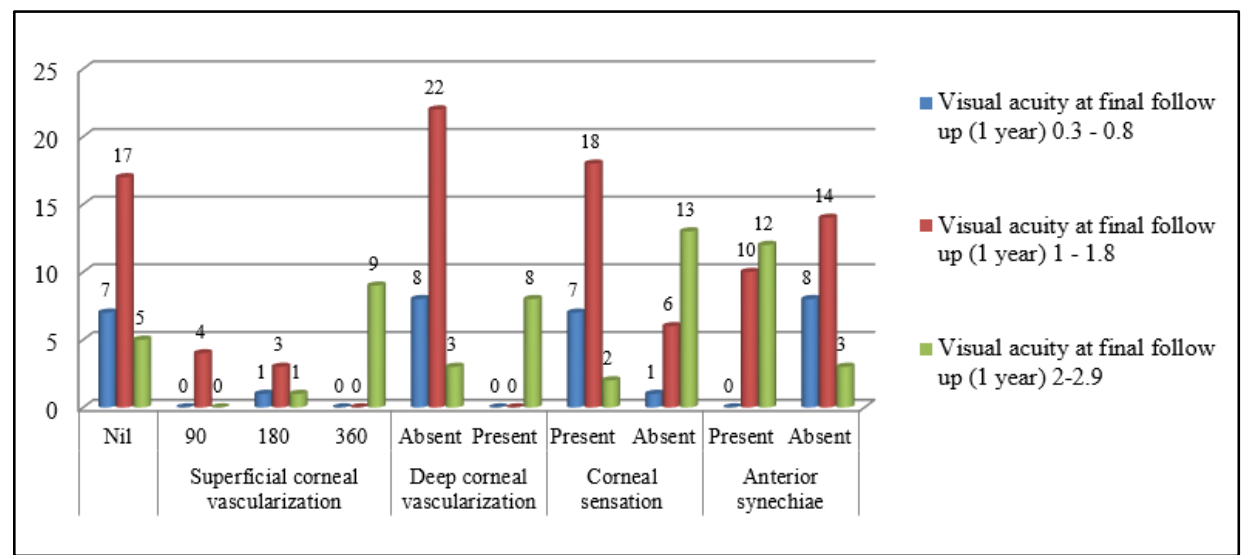

Fig. 5: Correlation between host risk factors and visual acuity at 1 year follow up ( $n=47)$

Fig. 5 demonstrates correlation of host risk factors with visual acuity at 1 year follow up. Chi square tests were executed to test the association between host factors (presence of superficial and deep vascularisation, anterior synechiae and absent corneal sensations) and estimated a positive association with a significant calculated $\mathrm{p}$ value $(\leq 0.01)$.

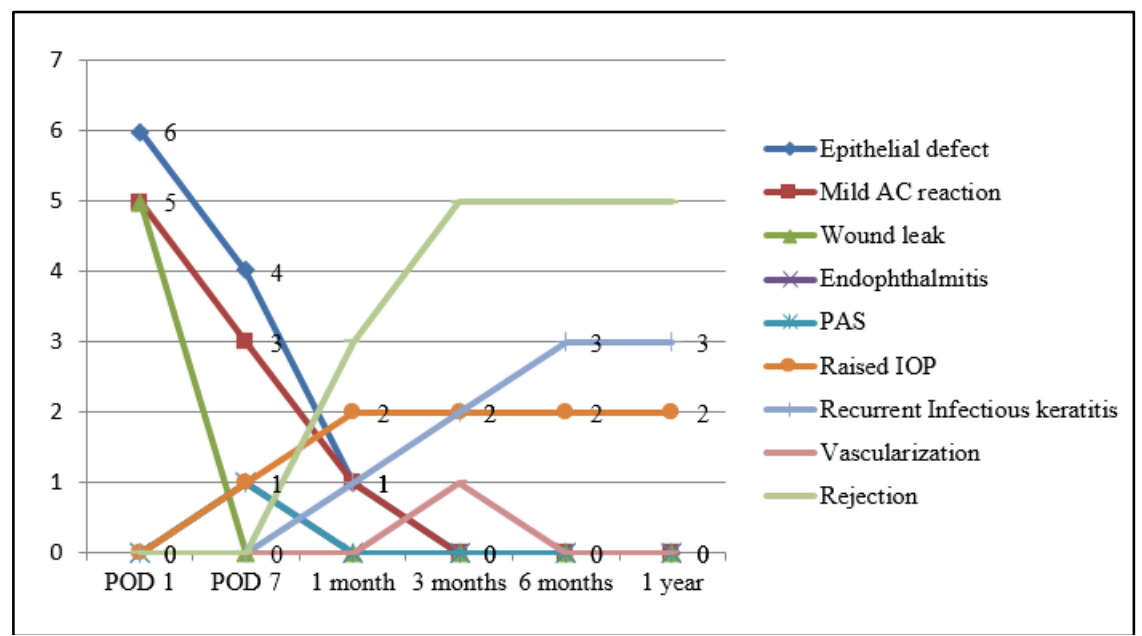

Fig. 6: Frequency distribution of complications at all follow ups $(\mathrm{n}=50)$ 
Fig. 6 shows the distribution of the post-operative complications of PK seen at all follow ups. At POD 1, the most common complication observed was epithelial defect, occurring in 6 of 50 recipients (12\%). Mild AC reaction was observed in $5(10 \%)$ recipients. Whereas, Graft rejection was observed as the most common complication at 3 months, 6 months and 1 year. At 1year follow up, 40 recipients (out of 47) didn't have any complications. 1 patient developed endophthalmitis.

\section{Discussion}

Penetrating keratoplasty is the most commonly performed allograft and can visually rehabilitate patients with corneal blindness. The prognosis depends on many variables, including etiology of corneal blindness in recipients, the donor cornea, the surgical technique, good post-operative care and early identification and management of complications.

The indication for PK in the present study was essentially for optical and therapeutic purpose. After analyzing the data with appropriate statistical indices, the determinants of the study were evaluated.

\section{Age and Gender}

The demographic profile is an important determinant of the study as it affects the final visual outcome of PK. In this study, PK was performed in various age groups. The mean age of recipients in the present study is 39.88 years \pm 2.9 years. (at 95\% Confidence interval). The reason for this could be, as this age belongs to the working middle aged adults who are more susceptible to occupational trauma. The observed male preponderance could be due to the behavioral and the social aspects in our society causing less reporting of cases of females as compared to males. The slight drift towards age group of 30-60 years indicates their more involvement in outdoor activities. Edwards et al (2002) observed that in his patients of penetrating keratoplasty, the average age of patients was 47.5 years with peak in fifth and eight decade. Shilpa Joshi, et al (2012) $)^{5}$ found in their study that mean age of the patients was $55.24 \pm 17.90$ years. They also observed a slight male preponderance.

\section{Host Pre-Operative Diagnosis}

The pre-operative corneal pathology is a major determinant of the final graft outcome. In the present study, the most common corneal pathology requiring PK was non-healing/ perforated corneal ulcer $(28 \%)$. The second most common corneal pathology was leucomatous corneal opacity, which was $13(26 \%)$ followed by adherent Leucoma. Our results were consistent with Nupur Gupta et al (2015) ${ }^{1}$ who found that ocular trauma, infectious keratitis and post-surgical bullous keratopathy are responsible for the major burden of corneal blindness in India.

In the present study, post-surgical pseudophakic bullous keratopathy was $4 \%$ among indications. This is in contrast with the study conducted by Rahman, F Carley et al (2009) ${ }^{4}$ who observed that bullous keratopathy constituted a major proportion $(22 \%)$ of all the corneal pathologies requiring
PK. This difference is explained by the fact that in developing countries like India, infectious causes of corneal opacities still top the list in corneal morbidity, requiring maximum number of PK.

\section{Complications of PK}

In the present study, it was observed that persistent epithelial defect and post operative uveitis were the most frequent complications in the early post operative period and secondary glaucoma and graft rejection in the late post operative period. Post keratoplasty glaucoma (PKG) is one of the challenging issues important for the survival of the graft. Its diagnosis and management are much more difficult than glaucoma. In this study, the diagnosis of PKG was made if IOP rise persisted after one month following PK in the presence of glaucomatous optic disc changes. Similar to our study, Kamal Dodia, et al $(2014)^{7}$ observed in their study that persistent epithelial defects, graft rejection mainly endothelial type late graft rejection, and secondary glaucoma were the most common complications found. Similarly, Tanuj Dada, et al (2008) ${ }^{8}$ stated that glaucoma following penetrating keratoplasty (PK) is one of the most common cause for irreversible visual loss and the second leading cause for graft failure after rejection. Stephen U, et al $(2000)^{9}$ concluded saying that early complications include wound leak, persistent epithelial defect, suture problems, filamentary keratopathy, elevated intraocular pressure, choroidal haemorrhage, hyphaema, microbial keratitis, endophthalmitis, whereas late complications were epithelial down growth, refractive error, Graft rejection and glaucoma.

Thus according to the present study and related studies it is concluded that secondary glaucoma, epithelial defects and graft rejection remain important complications following PK. The study highlights the necessity to evaluate all recipients of penetrating keratoplasty over a subsequent period of follow up for any signs of complications and to manage the complications as early as possible.

\section{Final BCVA as a Dependent Variable of Pre-op BCVA}

In the present study, linear regression analysis was applied to ascertain the correlation between visual acuity preoperatively and at 1 year follow up. A linear correlation was established between the two variables. As the final visual outcome depends on the pre-operative host risk factors which also determine the visual acuity pre-operatively, it can be established that pre-operative VA is a predictor of the VA attained post keratoplasty. It is hoped that a better analysis and understanding of the dependence of post op visual outcome on pre-operative VA will, in future, help in refining the criteria for patient selection and in guiding clinical practice.

\section{Effect of Host Factors on Final Visual Outcome}

In the present study, it was observed in eyes with absence of superficial vascularization, the percentage of good visual outcome was maximum. P. Vassileva et al $(2002)^{10}$ in their study stated that $52 \%$ of patients with graft failure had stromal vascularisation pre-operatively and vascularisation 
of different degrees in host cornea was found to be the most important risk factor for graft failure. Abdo Karim Tourkmani et al $(2017)^{11}$ applied multivariate logistic regression analysis in their study which showed a significant relationship between ocular hypertension, ocular inflammation, and corneal neovascularisation with graft failure at 1year. In the collaborative corneal transplant study (CCTS) (1992), ${ }^{12}$ it was concluded that the degree and depth of preoperative vascularization determine onset and severity of rejection. Thus in the present study it was concluded that presence of superficial and deep vascularisation in host cornea, presence of anterior synechiae and corneal hypo aesthesia or anesthesia result in poor visual acuity and graft clarity and thus a poorer outcome of PK. Therefore, a gross assessment of high risk cases of PK must be done preoperatively to predict outcome of the surgery.

\section{Summary and Conclusion}

The study was done to evaluate the visual outcome in patients undergoing penetrating keratoplasty in terms of visual acuity. Visual improvement was assessed at the end of 1 year after the surgery. Penetrating keratoplasty done for optical indications resulted in fairly good visual outcome. However contrary to previous reports, outcomes in therapeutic PK were comparable to that of optical PK with an insignificant difference which was attributed to poor visual potential in indications other than optical. Correlation between the preoperative host factors and the final graft outcome was evaluated in terms of final visual acuity. The presence superficial and deep vascularisation, anterior synechiae and corneal hypoaesthesia pre-operatively in the host cornea strongly correlated with a poorer graft outcome. Careful workup of recipients for corneal transplantation with reference to local and systemic risk factors is of utmost importance in the outcome of corneal transplantation with regards to graft survival.

However, there are a few limitations to the present study. The sample size was small which limits the use of statistical test. Few questions included in the VF 14 questionnaire were not applicable to the study population. Hence a modified validated questionnaire to assess accurately the improvement in quality of life of recipients from this part of the world would be more appropriate. from: http://restoresight.org/ wpcontent/uploads/2014/01/Medical-Standards-November2013.pdf

4. Rahman I, Huang MC, Carley F, Hillarby MC, Vasileiadis GT, Tullo A. The influence of donor and recipient factors in allograft rejection of the human cornea. [Internet]. Advances in pediatrics. U.S. National Library of Medicine; 2010 [cited 2018Aug19]. Available from: https://www.ncbi.nlm.nih.gov/pubmed/19373273

5. Joshi SA, Jagdale SS, More PD, Deshpande M. [Internet]. Advances in pediatrics. U.S. National Library of Medicine; 2012 [cited 2018Sep7]. Available from: https://www.ncbi.nlm.nih.gov/pmc/articles/PMC3263238/

6. Indications for Corneal Transplantation in New Zealand ... [Internet]. [cited 2018Aug26]. Available from:

http://www.academia.edu/ 23338105/Indications_for_Corneal_Transplantation_in_New_ Zealand_1991_1999

7. Dodia Kamal R, Shah Nirzari M, Chudasama Rajesh K. Incidence of increased intraocular pressure and factors associated with it after optical penetrating keratoplasty at secondary care centre, India. 2014;6(1):14-8.

8. Dada T, Aggarwal A, Minudath K B, Vanathi M, Choudhary S, Gupta V, Sihota R, Panda A. Post-penetrating keratoplasty glaucoma. Indian J Ophthalmol. 2008;56:269-77.

9. Stephen U. Complications after Penetrating Keratoplasty: International Ophthalmology Clinics [Internet]. LWW. Oxford University Press; [cited 2018Sep7]. Available from: https://journals.lww.com/internatophthalmology/Citation/2000/01000/Complications_After_Pen etrating_Keratoplasty.5.aspx

10. Vassileva P. Graft Failure after Keratoplasty [Internet]. Comptes Rendus de l'Academie Bulgare des Sciences, vol.56, p.4:111; 2003 [cited 2018Sep7]. Available from: http://adsabs.harvard.edu/ abs/2003CRABS..56d.111V

11. Tourkmani AK, Sánchez-Huerta V, De Wit G. Weighing of risk factors for penetrating keratoplasty graft failure: application of Risk Score System. Int J Ophthalmol. 2017;10(3):372-7. doi:10.18240/ijo.2017.03.08.

12. The collaborative corneal transplantation studies (CCTS ... [Internet]. [cited 2018Aug21]. Available from: https://www.ncbi.nlm.nih.gov/ pubmed/1417537

How to cite this article: Vaishali, Kumar K, Sharma B. Study of visual outcome in patients undergoing penetrating keratoplasty. Indian J Clin Exp Ophthalmol $2019 ; 5(3): 352-7$.

\section{Source of Funding: None.}

\section{Conflict of Interest: None.}

\section{References}

1. Gupta N, Tandon R, Gupta SK, Sreenivas V, Vashist P. [Internet]. Advances in pediatrics. U.S. National Library of Medicine; 2013 [cited 2018Aug19]. Available from: https://www.ncbi.nlm.nih.gov/pmc/ articles/PMC3831688/

2. Burden of Corneal Blindness in India - Europe PMC Article ... [Internet]. [cited 2018Aug19]. Available from: http://europepmc.org/ articles/PMC3831688

3. EBAA Medical Standards - Eye Bank Association of ... [Internet]. [cited 2018Aug19]. Available 